\title{
Human major group rhinoviruses downmodulate the accessory function of monocytes by inducing IL-10
}

\author{
Johannes Stöckl, ${ }^{1}$ Helga Vetr, ${ }^{2}$ Otto Majdic, ${ }^{1}$ Gerhard Zlabinger, ${ }^{1}$ Ernst Kuechler, ${ }^{2}$ \\ and Walter Knapp ${ }^{1}$ \\ ${ }^{1}$ Institute of Immunology, and \\ ${ }^{2}$ Institute of Biochemistry, University of Vienna, A-1090 Vienna, Austria \\ Address correspondence to: Johannes Stöckl, Institute of Immunology, University of Vienna, Borschkegasse 8a, \\ A-1090 Vienna, Austria. Phone: 431-4277-64935; Fax: 431-4277-9649; E-mail: Johannes.Stoeckl@univie.ac.at.
}

Received for publication May 4, 1999, and accepted in revised form August 10, 1999.

\begin{abstract}
Human rhinoviruses (HRVs) are the predominant cause of the common cold. Although this disease is per se rather harmless, HRV infection is considered to set the stage for more dangerous pathogens in vivo. Here we demonstrate that HRV-14, a member of the major group HRV family, can efficiently inhibit antigen-induced T-cell proliferation and T-cell responses to allogeneic monocytes. HRV-14 triggered a significant downregulation of MHC class II molecules on monocytes. Moreover, supernatants from monocytes cultured in the presence of HRV-14 strongly reduced the allogeneic T-cell stimulatory property of untreated monocytes and monocyte-derived dendritic cells (md-DCs), whereas Epstein Barr virus-transformed B-lymphoblastoid cells were not sensitive. Analysis of the supernatant revealed that HRV-14 induced the production of significant amounts of the immunosuppressive cytokine IL-10. The important T-cell stimulatory cytokine IL-12 or the proinflammatory cytokines IL- $1 \beta$ or TNF- $\alpha$ were not detected or were only minimally detected. Finally, monocytes pretreated with HRV-14 were greatly inhibited in their production of IL-12 upon stimulation with IFN$\gamma /$ LPS. These observations suggest that altered cytokine production in mononuclear phagocytes upon interaction with HRV downmodulates appropriate immune responses during the viral infection.
\end{abstract}

J. Clin. Invest. 104:957-965 (1999).

\section{Introduction}

Common colds induced by the human rhinovirus (HRV) occur worldwide (1). The frequent appearance of HRV, and its economic importance in terms of employee absenteeism, physician visits, and medication costs in industrial countries, make it a subject of primary importance (2). Despite this, the pathogenesis of HRV infection remains poorly understood $(1,3)$.

HRV primarily infects the ciliated epithelial cells of the upper respiratory tract (4). However, histological examinations of HRV-infected nasal epithelium demonstrated no obvious changes in the morphology or integrity of the nasal epithelium $(4,5)$. Instead, HRV infection is accompanied by a release of inflammatory mediators (3). In particular, proinflammatory cytokines including IL$1 \beta$, TNF- $\alpha$, IL-8, IL-6, and IL-11 (6-8) and the vasoactive peptides bradykinin and lysyl-bradykinin $(9,10)$ were found in nasal secretions of patients with colds. In addition, cytokine production was detectable in HRV-infected epithelial cells in vitro $(6-8,10-13)$. As a result, it is now considered that common cold symptoms result from an inflammatory "cytokine disease" (3).

Attracted by these mediators, inflammatory leukocytes, preferentially granulocytes and monocytes, are found to migrate to the site of $\operatorname{HRV}$ infection $(9,14,15)$. However, despite recruitment of invading leukocytes, appropriate immune responses appear to be hindered or dysregulated in the respiratory tract upon HRV infec- tion. This causes a well-documented disposition to bacterial infections leading to sinusitis, otitis media, bronchitis, pneumonia, and asthmatic exacerbations (16-24).

Here we demonstrate that interaction of immune cells with HRV-14, a member of the major group HRV family, efficiently inhibits antigen-specific T-cell responses by inducing IL-10 production in mononuclear phagocytes. IL-10 is a prominent immunosuppressive cytokine, which is critically involved in terminating inflammatory reactions. Owing to its anti-inflammatory properties, release of IL-10 might be responsible for the disturbed cellular immune responses during HRV infection.

\section{Methods}

Media, reagents, and chemicals. The cell culture medium RPMI-1640 (GIBCO BRL, Grand Island, New York, USA) supplemented with $2 \mathrm{mM}$ L-glutamine, 10\% FCS, $100 \mathrm{U} / \mathrm{mL}$ penicillin, and $100 \mu \mathrm{g} / \mathrm{mL}$ streptomycin was used in this study. The HRV-blocking reagent WIN 52035-2 (25) was a kind gift from the Sterling-Winthrop Research Institute (Rensselaer, New York) and was used at a final concentration of $5 \mu \mathrm{g} / \mathrm{mL}$. The superantigens staphylococcal enterotoxin A (SEA) and B (SEB) from Staphylococcus aureus were obtained from Serva (Heidelberg, Germany) and were used at a concentration of 10 $\mathrm{ng} / \mathrm{mL}$. Tetanus toxoid and purified protein derivative of Mycobacterium tuberculosis (PPD) were purchased from Connaught Laboratories (Willowdale, Ontario, Cana- 

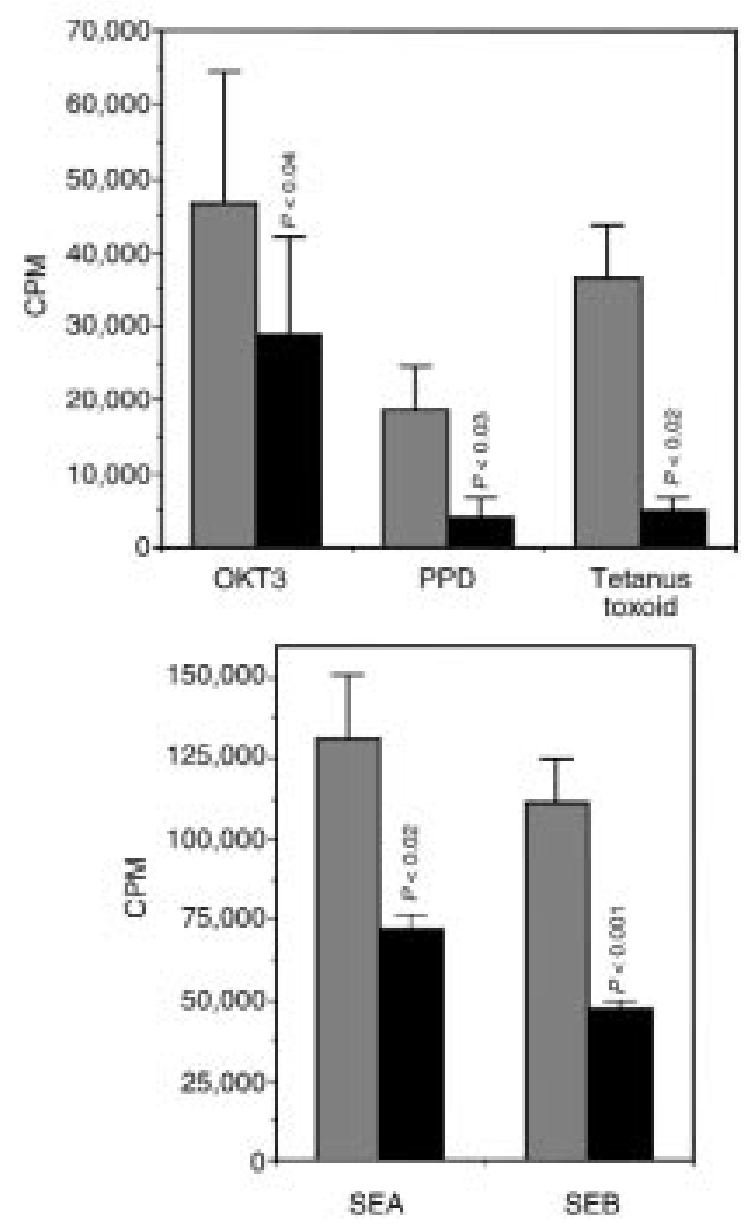

\section{Figure 1}

HRV-14 inhibits antigen-specific T-cell proliferation. PBMCs $\left(10^{5}\right)$ were stimulated with $\mathrm{mAb}$ OKT3 $(1 \mu \mathrm{g} / \mathrm{mL})$, SEA or SEB $(10 \mathrm{ng} / \mathrm{mL})$, and PPD or tetanus toxoid $(1 \mu \mathrm{g} / \mathrm{mL})$ in the presence (filled bars) or absence (gray bars) of HRV-14 (10 TCID 50 per cell). T-cell proliferation was measured on day 3 (for OKT3, SEA, SEB stimulation) and day 5 (for PPD, tetanus toxoid stimulation) of culture by adding (methyl- ${ }^{3} \mathrm{H}$ )-TdR followed by measuring thymidine incorporation 18 hours later. Background counts due to ongoing autologous MLR in the absence of specific stimulation were subtracted. The figure shows thymidine uptake, in cpm (mean \pm SEM) of 3 independent experiments. Paired Student's $t$ test was applied for statistical evaluation of data; corresponding $P$ values are indicated.

da) and used at a concentration of $1 \mu \mathrm{g} / \mathrm{mL}$. LPS from Escherichia coli (serotype 0127-B8) and polymyxin B were obtained from Sigma Chemie GmbH (Deisenhofen, Germany). Recombinant human GM-CSF and IL-4 were kindly provided by Novartis Research Institute (Vienna, Austria). IFN- $\gamma$ was a gift from G.R. Adolf (Ernst Boehringer Institut für Arzneimittelforschung, Vienna, Austria). IL-10 was purchased from R\&D Systems Inc. (Minneapolis, Minnesota, USA).

Antibodies used in this study. The following murine $\mathrm{mAb}$ 's were generated in our laboratory: negative control mAb VIAP (calf intestine alkaline phosphatase-specific), 6B6 (CD11a), VIM13 (CD14), 4D3 (CD33), and $1 / 47$ (MHC class II). Hybridomas producing $\mathrm{mAb}$
W6/32 (MHC class I), G28-5 (CD40), and TS2/9 (CD58) were obtained from American Tissue Culture Collection (ATCC; Rockville, Maryland, USA). UCHT-1 (CD3), MEM18 (CD14), and UCHL1 (CD45R0) were kindly provided by An der Grub (Bio Forschungs $\mathrm{GmbH}$, Kaumberg, Austria). OKT3 (CD3) was obtained from Ortho Diagnostics (Raritan, New Jersey, USA). The mAb HD37 (CD19) was provided by G. Moldenhauer (Heidelberg, Germany). MAb RR1/1 (CD54) was a gift from Bender AG (Vienna, Austria). The mAb L307 (CD80) was from Becton Dickinson Immunocytometry Systems (San Jose, California, USA). IT2.2 (CD86) and the PE-labeled mAb C11.5 (anti-IL-12) were purchased from PharMingen (San Diego, California, USA). MAb $3 \mathrm{G} 8$ (CD16), the FITC-labeled mAb MP9-20A4 (anti-TNF- $\alpha$ ), and PE-labeled mAb JES3-9D7 (anti-IL10) for cytoplasmic staining of IL-10 were obtained from Caltag Laboratories Inc. (Burlingame, California, USA). FITC-labeled FIB-3 mAb (anti-IL-1 $\beta$ ) was from Dianova (Hamburg, Germany). The neutralizing polyclonal anti-IL-10 antibody (PAL-hIL10) was obtained from Strahtmann Biotech (Hannover, Germany).

Rhinovirus preparation. HRV-14 was obtained from ATCC and routinely grown in suspension cultures of HeLa cells (strain Ohio; Flow Laboratories, McLean, Virginia, USA). Cells were cultivated in S-MEM medium (Joklik modification; catalog no. 22300-107; Life Technologies Inc., Rockville, Maryland, USA) supplemented with $7 \%$ heat-inactivated horse serum (catalog number 01051-M; Diagnostic Products GesmbLT, Wiener Neudorf, Austria), $1 \%$ penicillin/streptomycin, $1 \%$ glutamine, 1\% pluronic F68 (catalog no. P1300; Sigma Chemical Co., St. Louis, Missouri, USA), and 1\% nonessential amino acids. The same medium was used during infections, except that serum was reduced to $2 \%$ and $1 \mathrm{mM}$ $\mathrm{MgCl}_{2}$ was added. Cells were infected at a ratio of ten $50 \%$ tissue culture infectious doses (TCID) 50 per cell. HRV-14 was harvested 40 hours after infection. Cell debris was pelleted by low-speed centrifugation. HRV was prepared from cell culture supernatant by polyethanolglycol (PEG) precipitation (7\% PEG 6000, 3\% $\mathrm{NaCl}$ ). Virus was resuspended from the PEG pellet in PBS, aliquoted, and stored at $-70^{\circ} \mathrm{C}$. Working dilutions of used HRV-14 stock preparations contained less than $10 \mathrm{pg}$ LPS/mL. No biologic effects of these very low LPS concentrations were detectable. The preparations did not induce significant TNF- $\alpha$ or IL- 6 production in monocytes (see Figure 5). Moreover, the capacities of HRV-14 preparations to induce IL-10 production, inhibit T-cell proliferation, and downregulate MHC class II expression could not be inhibited by the LPS inhibitor polymyxin $B(10 \mu \mathrm{g} / \mathrm{mL})$ (data not shown).

Preparation of purified HRV. Virus stock preparations were further purified using sucrose gradients. Virus was pelleted by centrifugation at $100,000 \mathrm{~g}$ for 2 hours and resuspended in a small volume of "virus buffer" $(50 \mathrm{mM}$ Tris [ $\mathrm{pH} 7.5$ ], $2 \mathrm{mM} \mathrm{MgCl} 2$ ). It was then treated with DNase I (5 mg/mL; catalog no. 104169; Boehringer Mannheim, Mannheim, Germany) and RNase A (5 
a

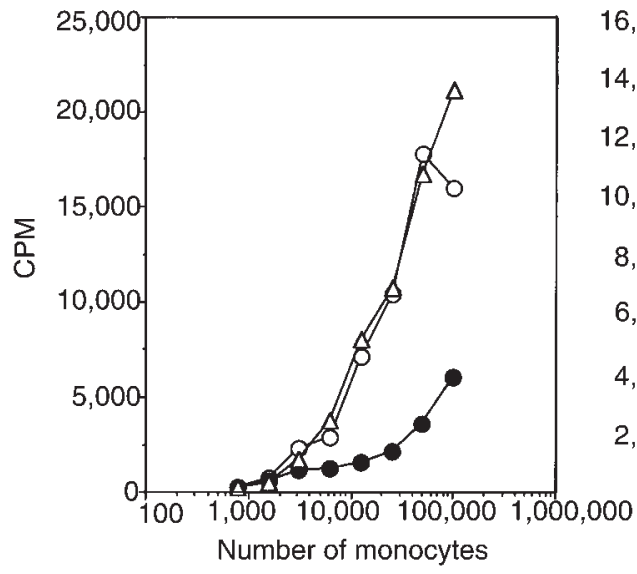

b

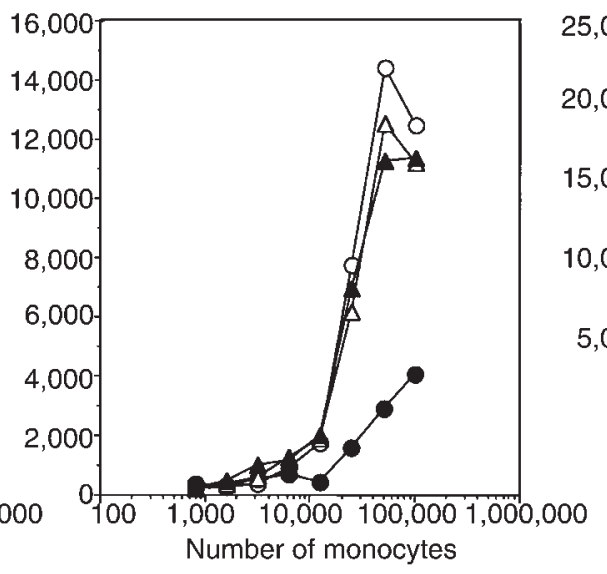

$c$

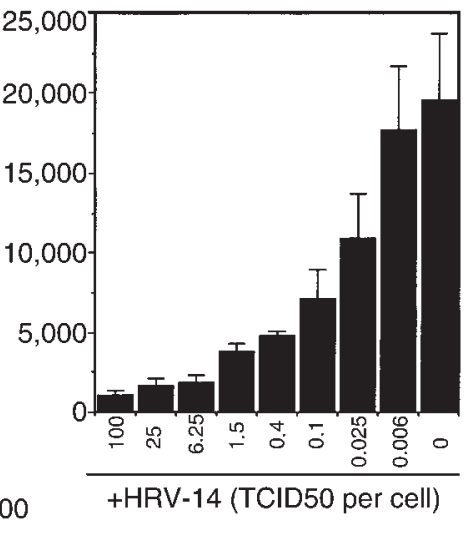

Figure 2

Inhibition of allogeneic T-cell proliferation by HRV-14. (a) Purified T cells $\left(10^{5}\right)$ were incubated with graded numbers of allogeneic, purified monocytes in the presence of HRV-14 (10 TCID 50 per cell; filled circles), control HeLa cell culture supernatants (open triangles), or medium alone (open circles). Proliferation of T cells was monitored on day 5 of culture by adding (methyl- ${ }^{3} \mathrm{H}$ )-TdR followed by measuring thymidine incorporation 18 hours later. The results shown are representative of 5 independent experiments. (b) Pretreatment of HRV-14 with WIN $52035-2$ ( $5 \mu \mathrm{g} / \mathrm{mL}$ ) for 30 minutes abolishes the inhibitory effect of HRV-14 (filled triangles). Addition of WIN $52035-2$ ( $5 \mu \mathrm{g} / \mathrm{mL}$ ) alone (open triangles) did not influence T-cell proliferation. The results are representative of 2 independent experiments. (c) Dose responses of HRV-14 particles on monocyte-induced allogeneic T-cell proliferation. The figure shows mean values \pm SEM of 3 experiments.

mg/mL; catalog no. 104159; Boehringer Mannheim) for 10 minutes at room temperature and further digested with trypsin (0.5 mg/mL; catalog no. 01104-H; Dipro) for 5 minutes at $37^{\circ} \mathrm{C}$. After addition of $N$-laurylsar$\operatorname{cosin}$ (0.1\%; catalog no. LA083; Schuchardt, Munich, Germany) digestion was continued at $4{ }^{\circ} \mathrm{C}$ overnight. After removal of unsoluble material by low-speed centrifugation, the sample was centrifuged on a sucrose gradient ( $7.5-45 \%$ sucrose in virus buffer) for 2 hours at $155,000 \mathrm{~g}$. Virus-containing fractions are visible as a turbid band in the middle of the gradient and were collected with a syringe. Virus was concentrated by pelleting and resuspension in virus buffer.

Ultraviolet inactivation of virus. Ultraviolet-inactivated (UV-inactivated) virus was prepared by irradiating virus suspensions in a 24-well tissue culture dish (200 $\mu \mathrm{L} /$ well) on ice for 10 minutes with a $75 \mathrm{~W}$ UV source $(254 \mathrm{~nm})$ at a distance of $5 \mathrm{~cm}$. Treatment resulted in complete loss of infectious titer.

Cell preparation. PBMCs were isolated from heparinized whole blood of normal healthy donors by standard density gradient centrifugation with Ficoll-Paque (Pharmacia, Uppsala, Sweden). Subsequently, T cells and monocytes were separated by magnetic sorting using the MACS technique (Miltenyi Biotec GmbH, Bergisch Gladbach, Germany) as described previously (26). Purified $T$ cells were obtained through negative depletion of CD11b, CD14, CD16, CD19, CD33, and MHC class II-positive cells with the respective mAb's. Monocytes were enriched by using the biotinylated CD14 mAb VIM13 (purity >95\%) as described previously (26).

Cultivation of monocytes. Monocytes $\left(1 \times 10^{6} / \mathrm{mL}\right)$ were cultured in 24-well plates (Corning-Costar Europe, Badhoevedorp, the Netherlands) in the presence of HRV-14
(10 TCID $_{50}$ per cell) or aliquot amounts of supernatants from uninfected $\mathrm{HeLa}$ cells at $37^{\circ} \mathrm{C}$. After 2 days, cells were harvested, washed in PBS, and analyzed by flow cytometry. Monocyte-derived dendritic cells (md-DCs) were generated by culturing purified blood monocytes for 8 days with a combination of GM-CSF $(50 \mathrm{ng} / \mathrm{mL})$ and IL-4 (100 U/mL) as described previously (26).

$T$-cell proliferation assays. For the allogeneic mixed lymphocyte reaction (MLR), allogeneic, purified T cells $\left(10^{5}\right)$ were incubated with graded numbers of irradiated (30 Gy, ${ }^{137} \mathrm{Cs}$ source) freshly isolated monocytes, mdDCs, or Epstein Barr virus-transformed (EBV-transformed) B-lymphoblastoid cells (B-LCLs) for the indicated periods. HRV (10 TCID $_{50}$ per cell) or respective aliquots of mock-cultured HeLa supernatants were added at the beginning of the MLR. In some experiments, supernatants $(100 \mu \mathrm{L})$ of monocytes cultured in the presence of HRV-14 or HeLa supernatants were added. Experiments were performed in 96-well cell culture plates in RPMI-1640 medium supplemented with $5 \%$ human AB-serum (PAA Laboratories, Munich, Germany). Proliferation of T cells was monitored by measuring (methyl- ${ }^{3} \mathrm{H}$ )-TdR (ICN Pharmaceuticals Inc., Irvine, California, USA) incorporation on day 5 of culture. Cells were harvested 18 hours later, and radioactivity was determined on a microplate scintillation counter (Packard, Meriden, Connecticut).

Antigen stimulation assays were performed with fresh PBMCs $\left(10^{5}\right.$ per well). The cultures were set up in the presence of tetanus toxoid $(1 \mu \mathrm{g} / \mathrm{mL})$, PPD (1 $\mu \mathrm{g} / \mathrm{mL})$, SEA or SEB $(10 \mathrm{ng} / \mathrm{mL})$, or OKT3 $(1 \mu \mathrm{g} / \mathrm{mL})$. Proliferation was measured by (methyl- $\left.{ }^{3} \mathrm{H}\right)$-TdR incorporation on day 3 (SEA, SEB, OKT3) and day 5 (tetanus toxoid, PPD) of culture. 


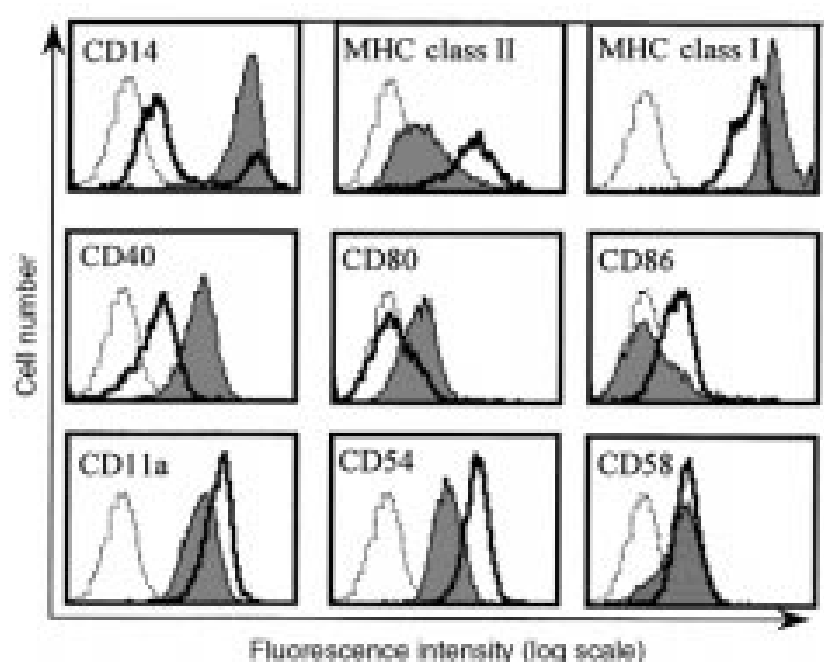

Figure 3

Expression of cell-surface molecules on HRV-14-treated monocytes. Purified monocytes were cultured in the presence of HRV-14 (10 $\mathrm{TCID}_{50}$ per cell; gray histograms) or control HeLa cell culture supernatant (thick line) for 2 days. Binding of specific mAb's was viewed by Oregon-Green-conjugated goat anti-mouse antibodies and analyzed by flow cytometry. The figure shows overlay histogram profiles including the irrelevant control mAb VIAP (dotted line) which are representative of 3 independent experiments.

Immunofluorescence analysis. For membrane staining, cells $\left(5 \times 10^{5}\right)$ were incubated for 30 minutes at $0-4^{\circ} \mathrm{C}$ with unconjugated $\mathrm{mAb}$. After washing twice with PBS, Oregon Green-conjugated goat anti-mouse antibody from Molecular Probes Inc. (Eugene, Oregon, USA) was used as a second-step reagent. Flow cytometric analysis was performed using a FACScan flow cytometer (Becton Dickinson).

Determination of cytokine production. Monocytes $(1 \times$ $10^{6} / \mathrm{mL}$ ) were cultured either mock treated or stimulated with LPS $(100 \mathrm{ng} / \mathrm{mL})$, with or without pretreatment for 24 hours with IFN- $\gamma(300 \mathrm{U} / \mathrm{mL})$, or in the presence of HRV (10 TCID 50 /monocyte) in 24-well plates (Corning-Costar Europe). After 24 hours, the supernatants were harvested and analyzed by ELISA or used in T-cell proliferation assays. For cytoplasmic staining, monensin $(5 \mu \mathrm{M})$ was added during the last 12 hours. The cells were harvested and fixed for 20 minutes at room temperature by adding $100 \mu \mathrm{L}$ of FIX solution (An der Grub). Subsequently, cells were washed once with $4 \mathrm{~mL}$ of PBS, resuspended in $100 \mu \mathrm{L}$ of PBS, and permeabilized by the addition of $100 \mu \mathrm{L}$ of PERM solution (An der Grub). Immediately, the indicated PE-conjugated anti-cytokine mAb's were added and incubated for 20 minutes at room temperature. The cells were then washed twice, resuspended in PBS $(200 \mu \mathrm{L})$, and analyzed by flow cytometry.

Cytokines were measured by sandwich ELISAs using matched-pair antibodies. Capture and detection antibodies for human IL-1 $\beta$ were obtained from Genzyme Pharmaceuticals (Cambridge, Massachusetts, USA); for
IL-10 and IL-12 p70, from R\&D Systems Inc.; and for TNF- $\alpha$, from PharMingen. Standards consisted of human recombinant material from R\&D Systems Inc. Assays were performed in duplicate according to the recommendations of the manufacturers. The lower limit of detection was $10 \mathrm{pg} / \mathrm{mL}$ for IL- $1 \beta$ and 20 $\mathrm{pg} / \mathrm{mL}$ for IL-10, IL-12, and TNF- $\alpha$.

\section{Results}

$H R V-14$ reduces antigen-induced T-cell proliferation. To analyze the influence of $\mathrm{HRV}$ on immune responses, we investigated its effect on antigen-induced T-cell proliferation. Results shown in Figure 1 demonstrate that addition of major group HRV-14 to PBMCs strongly reduced the proliferative $\mathrm{T}$-cell response induced by recall antigens (tetanus toxoid, $P P D$ ), superantigens (SEA, SEB), or CD3 mAb OKT3. In addition, T-cell proliferation induced by allogeneic monocytes was also strongly inhibited (Figure 2a). These effects were observed with purified HRV-14 and with UV-inactivated HRV-14 (data not shown) but were not induced with supernatants from uninfected HeLa cells used for control (Figure 2a). Preincubation of HRV-14 with WIN 52035-2, which sterically blocks the binding sites on HRV for its cellular receptor ICAM-1 (CD54) (25), reversed the inhibitory effect of HRV-14 (Figure 2b). Thus, binding of HRV-14 to cells was essential for inhibition of T-cell proliferation. This observation also demonstrates that direct viral effects and not contaminations are responsible. Titration of HRV-14 revealed that significant inhibitory effects were detectable down to an HRV-14 $\left(\mathrm{TCID}_{50}\right) /$ cell ratio of 0.025:1 (Figure 2c).

Downregulation of $M H C$ class II expression on monocytes upon culture with HRV-14. Stimulation of T cells by antigen-presenting cells is based on a complex molecular interaction that involves MHC-, adhesion-, and costimulatory molecules as well as cytokines. To elucidate which of these molecules might be affected by HRV-14, we cultured monocytes in the presence of HRV-14 or supernatants from uninfected HeLa cells for 2 days and analyzed the immunophenotype of the cells.

As can be seen in Figure 3, interaction of monocytes with HRV-14 had pronounced effects on their marker profile. The spontaneous downregulation of CD14 expression due to cultivation of monocytes was prevented in the presence of HRV-14. Surprisingly, the costimulatory molecules CD80 and CD40 were found to be neoexpressed or upregulated, respectively, on monocytes cultured in the presence of HRV-14 compared with mock-treated cells. Expression of the costimulatory molecule CD86 and the adhesion molecule CD54 was downregulated on monocytes upon HRV-14 interaction. Other important adhesion molecules such as CD11a and CD58 were only minimally affected. Most strikingly, however, cultivation of monocytes in the presence of HRV-14 strongly downregulated cell-surface expression of MHC class II molecules (Figure 3), in some instances down to $20 \%$ of control cells. In contrast, MHC class I expression was found to be slightly upregulated. 

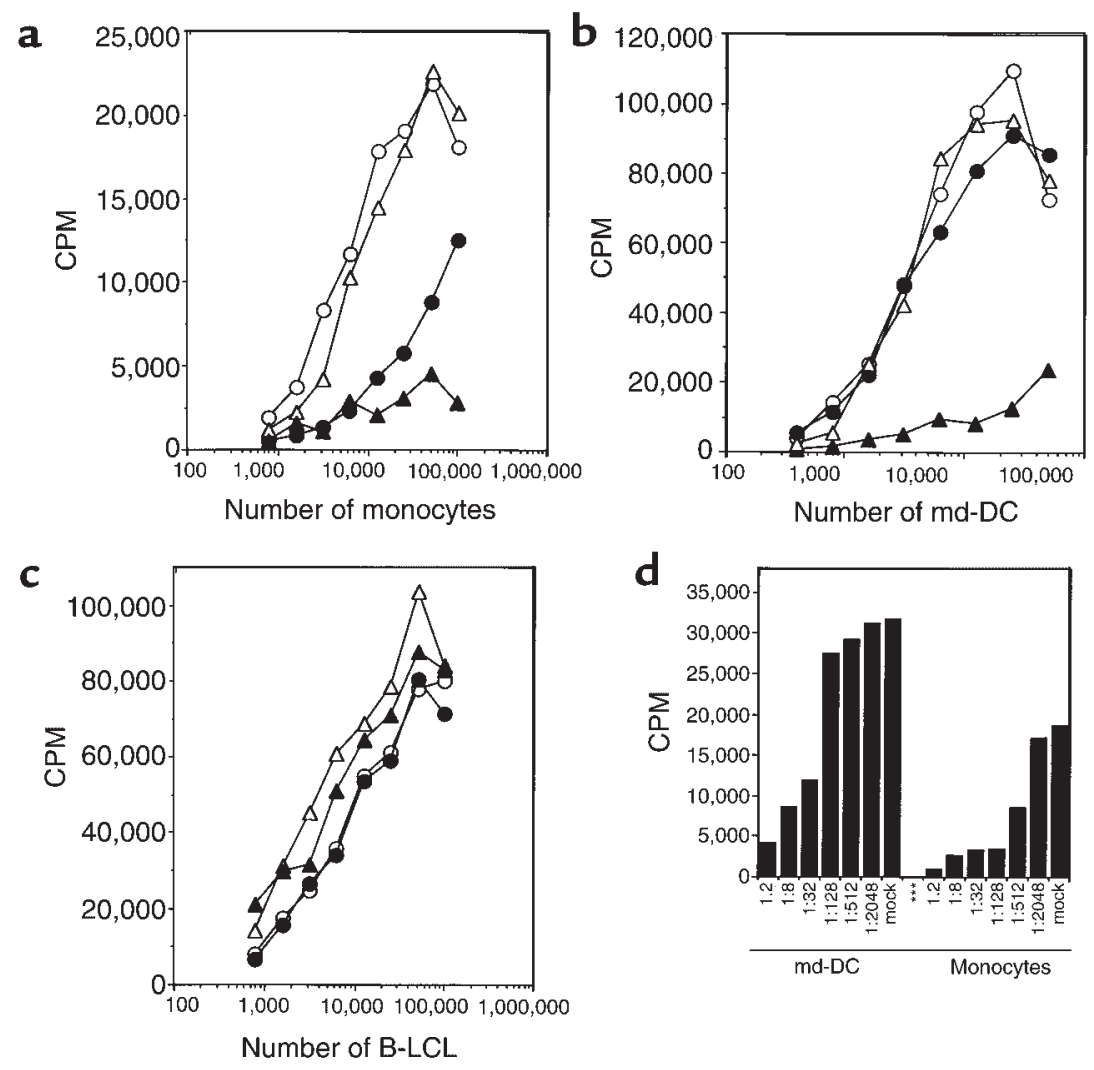

Figure 4

HRV-14-treated monocytes release an inhibitory factor. Monocytes were cultured for 2 days in the absence or presence of HRV-14 (10 TCID 50 per cell). The cell culture supernatants were harvested and examined in the allogeneic T-cell proliferation assay stimulated by irradiated monocytes (a), md-DCs (b), or B-LCLs (c). T-cell proliferative responses in the presence of supernatants of HRV-14-treated monocytes (filled triangles), mock-treated monocytes (open triangles), HRV-14 (filled circles), or medium alone (open circles) were tested in parallel. The figure shows results representative of 4 independent experiments. (d) Dose responses of decreasing amounts of supernatants obtained from HRV-14-treated monocytes were tested in the allogeneic T-cell proliferation assay stimulated by monocytes and md-DCs.
Monocytes produce an inhibitory factor upon interaction with HRV-14. The observations described here raised the question of how HRV-14 can downregulate the T-cell stimulatory capacity of monocytes. A first clue was obtained when the supernatant of monocytes cultured in the presence of HRV-14 for 2 days was transferred to untreated monocytes. Addition of this supernatant, but not of the supernatant of mock-treated monocytes, strongly reduced the proliferative $\mathrm{T}$-cell response induced by allogeneic monocytes (Figure 4a). Remarkably, inhibition by the supernatant of monocytes cultured in the presence of HRV-14 was always much stronger than the effect produced by addition of the virus alone and worked even when diluted down to 1:512 (Figure 4, a and d). Adding this supernatant to md-DCs, we found that the allostimulatory capacity of md-DCs was also strongly reduced, whereas HRV-14 alone showed no such effect (Figure 4, b and d). In contrast to monocytes and md-DCs, neither the supernatant of HRV-cultured monocytes nor the virus alone was able to diminish the allogeneic T-cell response induced by EBV-transformed B-LCLs (Figure 4c).

$H R V-14$ induces IL-10 production in monocytes. A prominent immunosuppressive factor that strongly inhibits the accessory function of monocytes and of DCs (27) and downregulates MHC class II cell-surface expression (28) but does not inhibit B cell-mediated responses $(27,28)$ is the cytokine IL-10. Therefore, we analyzed the supernatant of HRV-14-treated monocytes after 2 days of culture for the presence of IL-10. The results of this analysis revealed that large amounts of IL-10 were present in the supernatant of HRV-14-treated monocytes (Figure 5a). The amounts of IL-10 and the number of monocytes producing this cytokine (Figure 5b) upon stimulation with HRV-14 were comparable with those seen after LPS stimulation. HRV-14 samples used in this study contained no detectable IL-10 (<20 pg/mL) and low LPS contamination $(<10 \mathrm{pg} / \mathrm{mL})$. Moreover, in contrast to LPS stimulation, HRV-14 did not induce production of the proinflammatory cytokine TNF- $\alpha$ and induced only small amounts of IL-1 $\beta$ in monocytes (Figure 5a).

$H R V-14$ inhibits IL-12 production in monocytes. An important immunoregulatory property of IL-10 is its ability to inhibit the production of IL-12 (29), the critical cytokine that drives $\mathrm{T}$-cell responses toward type 1 effector cells (30). This prompted us to examine the influence of HRV-14 on IL-12 production of monocytes. Monocytes are known to produce IL-12 upon stimulation by IFN- $\gamma /$ LPS (31). In contrast, pretreatment of monocytes with IFN- $\gamma$ and subsequent addition of HRV-14 did not result in IL-12 production (Figure 6a). We therefore asked whether HRV-14 might inhibit the induction of IL-12. For this purpose, monocytes were cultured with HRV-14 or control HeLa supernatants before IFN- $\gamma /$ LPS was added to induce IL-12 production. Pretreatment of monocytes with HRV-14 strongly inhibited the amounts of IL-12 (Figure 6a) and the number of cells producing IL-12 as a result of IFN- $\gamma /$ LPS stimulation, whereas IL-6 production was not reduced (Figure 6b). Inhibition of IL-12 production induced by HRV-14 was only partially reversed in the presence of neutralizing 


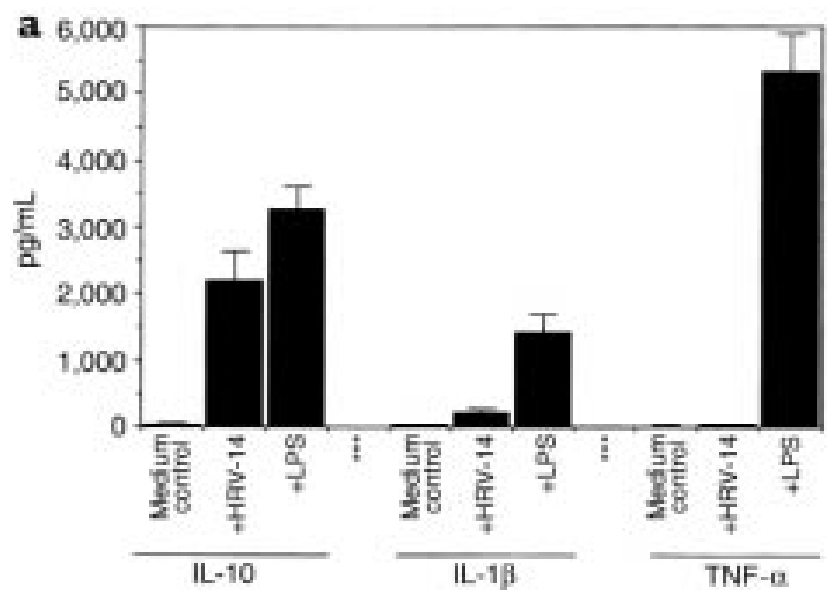

b

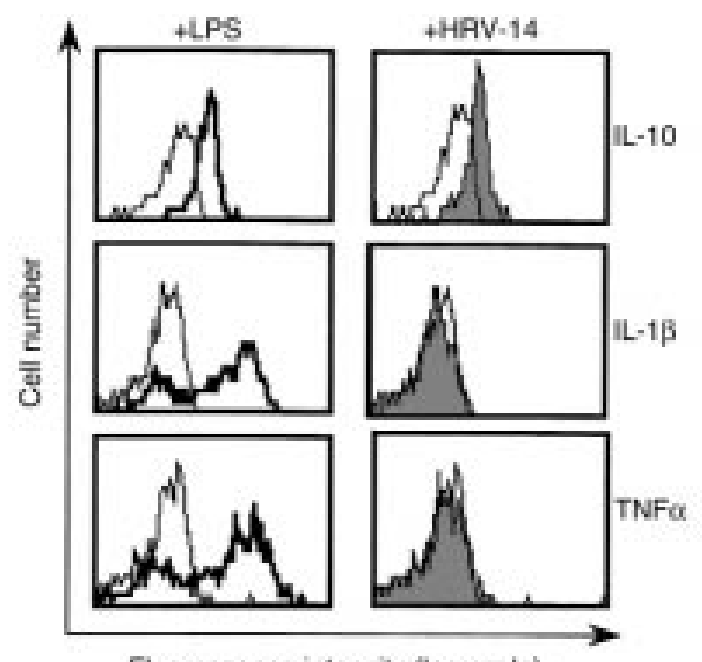

Fluorescence intensity (log scale)

Figure 5

Induction of IL-10 production in monocytes by HRV-14. (a) Supernatants of monocytes $\left(1 \times 10^{6} / \mathrm{mL}\right)$ cultured in the presence of HRV14 (10 TCID 50 per cell), LPS $(100 \mathrm{ng} / \mathrm{mL})$, or medium for 2 days were analyzed for IL-10, IL-1 $\beta$, and TNF- $\alpha$ production by ELISA. The figure shows mean values \pm SEM of 3 experiments. (b) In separate experiments, detection of cytokine production in monocytes was performed by cytoplasmic staining with specific mAb's. Monocytes $\left(1 \times 10^{6} / \mathrm{mL}\right)$ were cultured for 24 hours in the presence of HRV-14 (10 TCID 50 per cell) or LPS (100 ng/mL). After 12 hours of culture, monensin $(5 \mu \mathrm{M})$ was added in all instances. The cells were harvested, fixed, permeabilized, and subsequently stained with PE-labeled anti-IL-10 mAb. For determination of IL- $1 \beta$ or TNF- $\alpha$ production by cytoplasmic staining, monocytes were incubated with the indicated stimuli for 6 hours in the presence of monensin $(5 \mu \mathrm{M})$. Cytoplasmic cytokine expression was analyzed by flow cytometry. Overlay histograms show expression in mock-treated cells (open histograms) and monocytes stimulated with HRV-14 (gray histograms) or LPS (open histograms, thick line). The results shown are representative of 3 experiments.

anti-IL-10 antibodies (Figure 6b). This observation demonstrates that an additional IL-10-independent mechanism is critically involved in the selective inhibition of IL-12 production by HRV-14.

Neutralization of IL-10 abrogates the inbibitory effect of $H R V-14$. To ascertain that IL-10 was the responsible sup- pressive factor present in the supernatant of HRV14-treated monocytes, the supernatant was pretreated with neutralizing IL-10 antibodies and subsequently tested in the allo-MLR assay. Results in Figure 7 demonstrate that the addition of anti-IL-10 antibodies reversed the inhibitory effect of the supernatant obtained from HRV-14-treated monocytes and that monocytes stimulated $\mathrm{T}$ cells in the presence of this supernatant nearly as well as untreated cells. Because intact HRV-14 is still present in the supernatant obtained from HRV-14 treated monocytes, neutralizing anti-IL-10 antibodies did obviously inhibit both transferred IL-10 and IL-10 induced by transferred HRV-14.

\section{Discussion}

In this study, we demonstrate that HRV-14, a member of the major group HRV family, can efficiently inhibit antigen-specific T-cell responses. These inhibitory effects were found to result from a particular spectrum of cytokines released by mononuclear phagocytes upon HRV-14 interaction. Most prominent among them is IL-10, a well-established immunosuppressive cytokine (32), which is strongly induced by HRV-14. In contrast, major proinflammatory cytokines such as TNF- $\alpha$ and IL-1 $\beta$ and the Th1-activator IL-12 (30) are not induced or are only minimally induced, and IL-12 production induced by IFN- $\gamma /$ LPS was significantly inhibited in monocytes after HRV-14 interaction. On the basis of these observations, it is tempting to speculate that the particular cytokine profile induced by HRV in mononuclear phagocytes might have adverse effects on local immunity in areas of HRV infection. Such reduced local immunocompetence may predispose affected individuals to secondary infections and could possibly explain the frequently observed occurrence of sinusitis, otitis media, bronchitis, and pneumonia in HRV-infected persons $(23,24)$.

Inhibition of antigen-specific T-cell responses by major group HRV have been reported previously (33). This property cannot be attributed to cytopathic effects because HRV does not infect or damage leukocytes (34). Inhibition was explained by the capacity of major group HRV to bind the T-cell ligand ICAM-1 on accessory cells (monocytes) and thereby prevent accessory-cell to T-cell contact and, consequently, T-cell activation. Such a mechanism seems to be plausible, as other studies have shown that binding of HRV to ICAM-1 blocks ICAM-1/LFA-1 pair formation $(35,36)$. Although direct interference may contribute to a reduction of T-cell reactivity, it cannot account for the inhibitory effects of HRV observed by us. In particular, HRV-14 did not inhibit md-DCs or B-LCL-induced allogeneic T-cell responses, in which ICAM-1/LFA-1 interactions are also critically involved (37-39). Rather, the induction of a soluble factor by HRV-14 in monocytes seems to be primarily responsible for the inhibition of the antigen-specific T-cell response. Data presented here demonstrate that this factor is IL-10. First, addition of supernatant from HRV-14-treated mono- 
cytes only inhibited the allostimulatory capacity of monocytes and md-DCs, whereas B-LCLs were not affected, which is typical for IL-10 $(27,28)$. Second, monocytes stimulated with HRV-14 were found to produce biologically relevant amounts of IL-10 (27). Third, neutralization of IL-10 with a specific antibody reverted the blocking effects of HRV on T-cell stimulation.

The mechanism of inhibition of antigen-specific, cellular immune responses by IL-10 is well defined (32). Most relevant in this respect is the massive downregulation of MHC class II molecule expression in the presence of IL-10 (28). Accordingly, HRV-14-induced downregulation of MHC class II molecules was inhibited in the presence of anti-IL-10 antibodies (data not shown). In contrast to MHC class II, MHC class I expression on monocytes is only slightly affected by IL-10, and responsiveness of activated $\mathrm{CD}^{+} \mathrm{T}$ cells is even enhanced by IL-10 $(40,41)$. It has also been demonstrated that IL-10 reduces $\mathrm{CD} 80$ and $\mathrm{CD} 86$ costimulatory molecule expression $(41,42)$ and inhibits IL-12 production (29). Upon HRV-14 treatment of monocytes, we have also observed downregulation of CD86 expression and an inhibition of IL-12 production. However, inhibition of IL-12 production induced by HRV-14 was only partially reversed in the presence of neutralizing anti-IL-10 antibodies, indicating that an IL-10-independent mechanism is also critically involved, which is reminiscent of what has been found to be the case with measles viruses (31). Also in contrast to IL-10 effects, CD80 and CD40 expression was clearly upregulated in HRV-treated monocytes. Another marker reproducibly found to be upregulated on monocytes upon HRV-14 stimulation was CD14, a cell-surface molecule that is not significantly influenced by IL-10 $(40,42)$. Thus, the immunophenotype of HRV-14-treated monocytes differs in some aspects from that reported for IL-10 stimulation, and not all functional effects of HRV-14 may be due to induction of IL-10 production.

The classical cellular receptor for major group HRV is ICAM-1 $(43,44)$. Here we provide 3 pieces of evidence that engagement of ICAM-1 is required to elicit the inhibitory effects of HRV. First, cell-surface expression of ICAM-1 on monocytes was reduced upon HRV-14 interaction. This is likely to be primarily due to receptor usage of HRV-14 (45) and only marginally reversed in the presence of anti-IL-10 antibodies (data not shown). Second, the specific inhibitor WIN 52035-2, which blocks HRV binding to ICAM-1 (25), abolished the HRV-induced inhibitory capacity. Third, inhibition was not observed with the minor group HRV-2, which interacts with cells via the LDL-receptor and/or LDLreceptor-related proteins (46) (data not shown).

The mechanism of IL-10 induction in monocytes by HRV-14 and the role of ICAM-1 are not yet understood. Signal transduction via ICAM-1 is possible. It can be induced by specific antibodies $(47,48)$ or by its ligand fibrinogen $(49,50)$. We observed that engagement of ICAM-1 with CD54 mAb RR1/1 did not stimulate IL-10 production in monocytes (data not
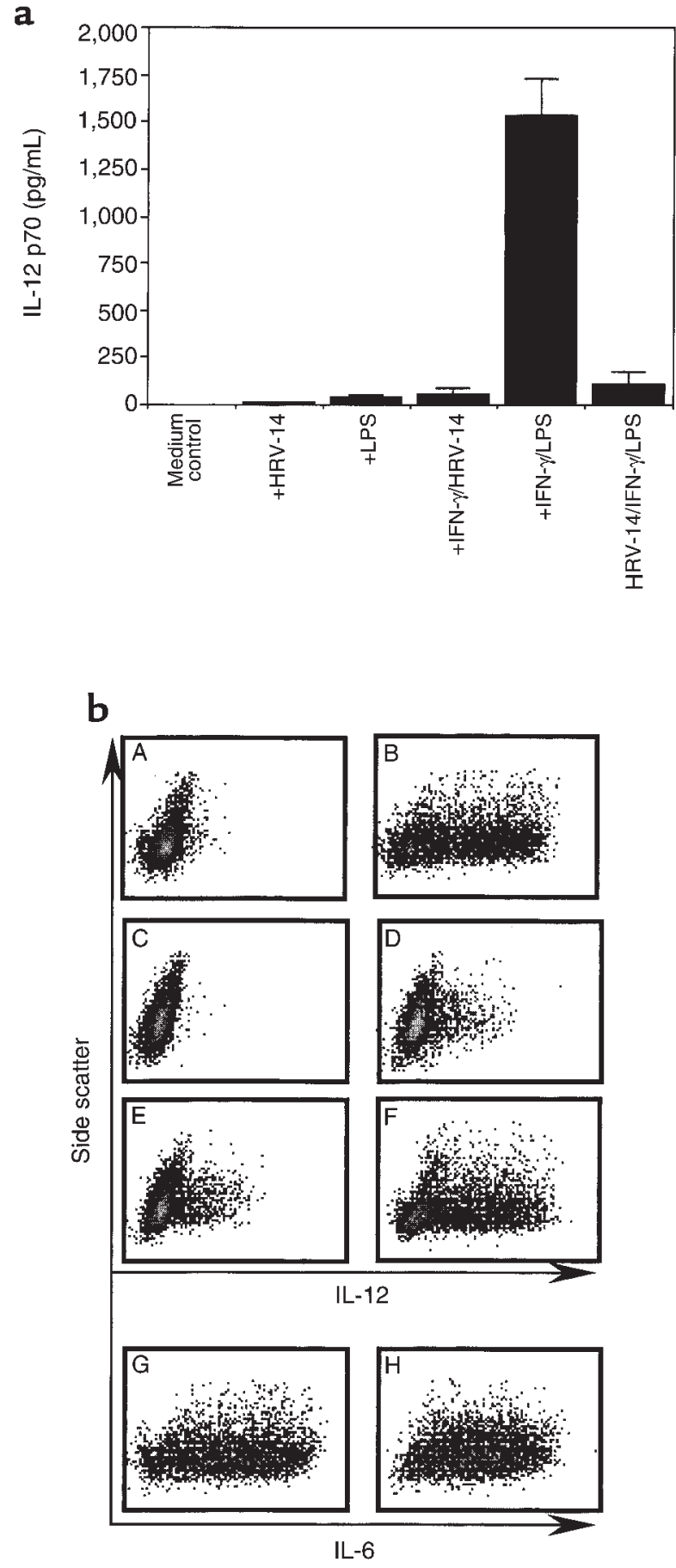

(Fluorescence intensity, log scale)

\section{Figure 6}

Inhibition of IL-12 production by HRV-14. Purified monocytes $(1 \times$ $10^{6} / \mathrm{mL}$ ) were cultured for 2 days in the absence or presence of HRV$14\left(10 \mathrm{TCID}_{50}\right.$ per cell) or LPS $(100 \mathrm{ng} / \mathrm{mL})$ with or without prestimulation with IFN- $\gamma(300 \mathrm{U} / \mathrm{mL})$ for 24 hours. Supernatants of cultured monocytes were analyzed for IL-12 p70 release by ELISA (a). The figure shows mean values \pm SEM of 3 experiments. (b) Cytoplasmic staining of monocytes with PE-conjugated anti-IL-12 mAb or anti-IL-6 mAb. Monocytes were stimulated as follows: mock stimulated $(\mathrm{A})$; +IFN- $\gamma /$ LPS (B and G); +HRV-14/IFN- $\gamma /$ LPS $(\mathrm{C}$ and $\mathrm{H})$; +HRV-14/anti-IL-10 antibody/IFN- $\gamma /$ LPS (D); +IL-10 (5 ng/mL)/IFN$\gamma /$ LPS (E); and +IL-10/anti-IL-10 antibody/IFN- $\gamma /$ LPS (F). 


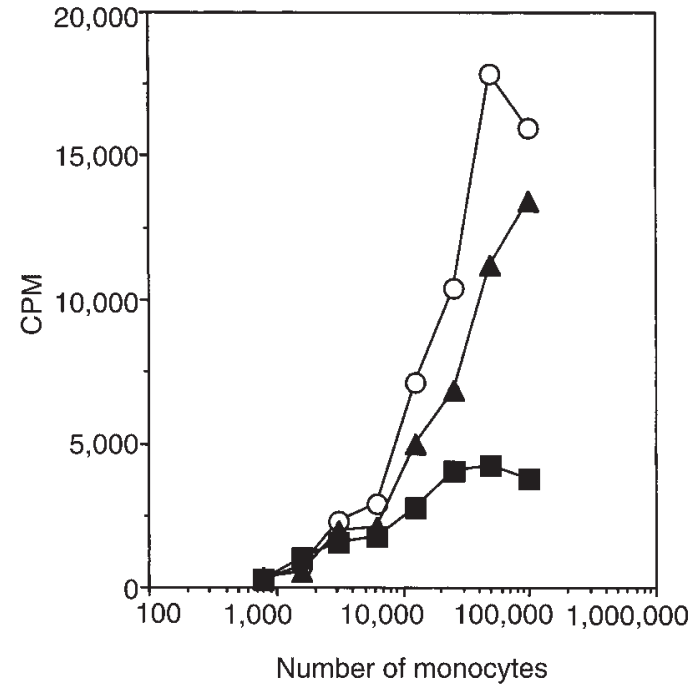

Figure 7

Neutralization of IL-10 abolishes the inhibitory effect of HRV-14. Purified T cells $\left(10^{5}\right)$ were incubated with graded numbers of allogeneic, purified monocytes in the absence (open circles) or presence of supernatants from HRV-14-treated monocytes that had been pretreated with (filled triangles) or without (filled squares) neutralizing anti-IL-10 antibodies.

shown). However, because $1 \mathrm{HRV}$ capsid has 60 receptor binding sites and binds to ICAM-1 with high avidity (51), rhinovirus particles might trigger pronounced cross-linking of ICAM-1 and subsequent signal transduction, leading to IL-10 production. It is conceivable that this may not be achieved by mAb's or by other ligands of ICAM-1. Gern and coworkers have recently reported that binding of HRV-16, another major group HRV, to airway macrophages, and induction of TNF- $\alpha$, were not inhibited with antibodies against ICAM-1 (34). These observations suggest that HRV may also interact with mononuclear phagocytes via yet undefined signal transducing receptor structures. Therefore, future experiments are necessary to clarify whether such cellular receptors for HRV are expressed on mononuclear phagocytes.

IL-10 is 1 of the key factors that regulate inflammatory responses $(32,52)$. The anti-inflammatory action of IL-10 is primarily achieved by inhibition of the production of proinflammatory cytokines such as IL-1, IL-6, and TNF- $\alpha$ (53-55). The crucial role of IL-10 in controlling inflammatory responses is best illustrated in IL-10 knockout mice that suffer from chronic inflammatory bowel disease (56) and in studies in which injection of IL-10 rescued mice from LPSinduced toxic shock (57). Common cold symptoms are considered to be caused by proinflammatory cytokines produced in the nasal mucosa (3). In the course of the infection, however, major group HRV may induce the production of IL-10 in infiltrating monocytes. This may result in a decrease of the local inflammatory reaction and in the inhibition of cellular immune responses.

\section{Acknowledgments}

The authors thank S. Künig, L. Gschwantler, I. Gösler, and $\mathrm{K}$. Wenhardt for expert technical assistance, and $\mathrm{M}$. Epstein, W. Pickl, and M. Waclavicek for critical reading of the manuscript. This work was supported by the Fonds zur Förderung der Wissenschaftlichen Forschung in Österreich (SFB 005).

1. Couch, R. 1996. Rhinoviruses. In Fields virology. B. Fields et al., editors. Lippincott-Raven Publishers. Philadelphia, PA. 713-734.

2. Garibaldi, R. 1985. Epidemiology of community-acquired respiratory tract infections in adults. Am. J. Med. 78:32-37.

3. Pitkäranta, A., and Hayden, F.G. 1998. What's new with common colds? Pathogenesis and diagnosis. Infect. Med. 15:50-59.

4. Turner, R.B., Hendley, J.O., and Gwaltney, J.M., Jr. 1982. Shedding of infected ciliated epithelial cells in rhinovirus colds. J. Infect. Dis. 145:849-853.

5. Winther, B., Brofeldt, S., Christensen, B., and Mygind, N. 1984. Light and scanning electron microscopy of nasal biopsy material from patients with naturally acquired common colds. Acta Otolaryngol. (Stockh.) 97:309-318.

6. Noah, T.L., et al. 1995. Nasal cytokine production in viral acute upper respiratory infection of childhood. J. Infect. Dis. 171:584-592.

7. Einarsson, O., Geba, G.P., Zhu, Z., Landry, M., and Elias, J.A. 1996. Interleukin 11: stimulation in vivo and in vitro by respiratory viruses and induction of airways hyperresponsiveness. J. Clin. Invest. 97:915-924.

8. Zhu, Z., et al. 1996. Rhinovirus stimulation of interleukin- 6 in vivo and in vitro. Evidence of nuclear factor $\kappa \mathrm{B}$-dependent transcriptional activation. J. Clin. Invest. 97:421-430.

9. Naclerio, R.M., et al. 1987. Kinins are generated during experimental rhinovirus colds. J. Infect. Dis. 157:133-142.

10. Proud, D., Naclerio, R.M., Gwaltney, J.M., and Hendley, J.O. 1990. Kinins are generated in nasal secretions during natural rhinovirus colds. J. Infect. Dis. 161:120-123.

11. Proud, D., et al. 1994. Increased levels of interleukin-1 are detected in nasal secretions of volunteers during experimental rhinovirus colds. J. Infect. Dis. 169:1007-1013.

12. Subauste, M.C., Jacoby, D.B., Richards, S.M., and Proud, D. 1995. Infection of a human respiratory epithelial cell line with rhinovirus. Induction of cytokine release and modulation of susceptibility to infection by cytokine exposure. J. Clin. Invest. 96:549-557.

13. Johnstone, S.L., et al. 1998. Low grade rhinovirus infection induces a prolonged release of IL-8 in pulmonary epithelium. J. Immunol. 160:6172-6181.

14. Winther, B., et al. 1984. Histopathologic examination and enumeration of polymorphonuclear leukocytes in the nasal mucosa during experimental rhinovirus colds. Acta Otolaryngol. (Stockh.) 413:19-24.

15. Levandowski, R.A., Weaver, C.W., and Jackson, G.G. 1988. Nasal-secretion leukocyte populations determined by flow cytometry during acute rhinovirus infection. J. Med. Virol. 25:423-432.

16. Evans, F.O., Jr., et al. 1975. Sinusitis of the maxillary antrum. N. Engl. J. Med. 293:735-739.

17. Elkhatieb, A., Hipskind, G., Woerner, D., and Hayden, F.G. 1993. Middle ear abnormalities during natural rhinovirus colds in adults. J. Infect. Dis. 168:618-621.

18. Buchman, C.A., Doyle, W.J., Skoner, D., Fireman, P., and Gwaltney, J.M., Jr. 1994. Otologic manifestations of experimental rhinovirus infection. Laryngoscope. 104:1295-1299.

19. Pitkäranta, A., Arruda, E., Malmberg, H., and Hayden, F.G. 1997. Detection of rhinovirus in sinus brushings of patients with acute community-acquired sinusitis by reverse transcription-PCR. J. Clin. Microbiol. 35:1791-1793.

20. Pitkäranta, A., Virolainen, A., Jero, J., Arruda, E., and Hayden, F.G. 1998. Detection of rhinovirus, respiratory syncytial virus and coronavirus infections in acute otitis media by reverse transcriptase polymerase chain reaction. Pediatrics. 102:291-295.

21. Pitkäranta, A., Jero, J., Arruda, E., Virolainen, A., and Hayden, F.G. 1998. Polymerase chain reaction-based detection of rhinovirus, respiratory syncytial virus and coronavirus in otitis media with effusion. J. Pediatr. 133:390-394.

22. Pitkäranta, A., and Hayden, F.G. 1998. Rhinoviruses: important respiratory pathogens. Ann. Med. 30:529-537.

23. Pitkäranta, A., and Hayden, F.G. 1998. What's new with common colds? Complications and management. Infect. Med. 15:117-128.

24. Gern, J.E., and Busse, W.W. 1999. Association of rhinovirus infections with asthma. Clin. Microbiol. Rev. 12:9-18.

25. Shepard, D.A., Heinz, B.A., and Rueckert, R.R. 1993. WIN 52032-2 inhibits both attachment and eclipse of human rhinovirus 14. J. Virol. 67:2245-2254.

26. Pickl, W.F., et al. 1996. Molecular and functional characteristics of dendritic cells generated from highly purified $\mathrm{CD} 14^{+}$peripheral blood 
monocytes. J. Immunol. 157:3850-3859.

27. Caux, C., et al. 1994. Interleukin 10 inhibits $\mathrm{T}$ cell alloreaction induced by human dendritic cells. Int. Immunol. 6:1177-1185.

28. de Waal Malefyt, R., et al. 1991. Interleukin 10 (IL-10) and viral IL-10 strongly reduce antigen-specific human $\mathrm{T}$ cell proliferation by diminishing the antigen presenting capacity of monocytes via downregulation of class II major histocompatibility expression. J. Exp. Med. 174:915-924.

29. D'Andrea, A., et al. 1993. Interleukin 10 inhibits human lymphocyte interferon gamma-production by suppressing natural killer cell stimulatory factor/IL-12 synthesis in accessory cells. J. Exp. Med. 178:1041-1048.

30. Trinchieri, G. 1993. Interleukin-12 and its role in the generation of Th1 cells. Immunol. Today. 14:335-338.

31. Karp, C.L., et al. 1996. Mechanism of suppression of cell-mediated immunity by measles virus. Science. 273:228-231.

32. de Waal Malefyt, R. 1998. Interleukin-10. In Cytokines. A. Mire-Sluis and R. Thorpe, editors. Academic Press. London, United Kingdom. 151-167.

33. Gern, J.E., Joseph, B., Galagan, D.M., Borcherding, W.R., and Dick, E.C. 1996. Rhinovirus inhibits antigen-specific $\mathrm{T}$ cell proliferation through an intercellular adhesion molecule-1-dependent mechanism. J. Infect. Dis. 174:1143-1150.

34. Gern, J.E., et al. 1996. Rhinovirus enters but does not replicate inside monocytes and airway macrophages. J. Immunol. 156:621-627.

35. Staunton, D.E., Dustin, M.L., Erickson, H.P., and Springer, T.A. 1990 The arrangement of the immunoglobulin-like domains of ICAM-1 and the binding sites for LFA-1 and rhinovirus. Cell. 61:243-254.

36. Piela-Smith, T.H., Aneiro, L., and Korn, J.H. 1991. Binding of human rhinovirus and $\mathrm{T}$ cells to intercellular adhesion molecule- 1 on human fibroblasts. Discordance between effects of IL-1 and IFN- $\gamma$. J. Immunol. 147:1831-1836

37. Sanders, V.M., Snyder, J.M., Uhr, J.W., and Vitetta, E.S. 1986. Characterization of the physical interaction between antigen-specific $\mathrm{B}$ and $\mathrm{T}$ cells. J. Immunol. 137:2395-2404.

38. King, P.D., and Katz, D.R. 1989. Human tonsillar dendritic cell-induced $T$ cell responses: analysis of molecular mechanisms using monoclonal antibodies. Eur. J. Immunol. 19:581-587.

39. Metlay, J.P., Pure, E., and Steinman, R.M. 1989. Distinct features of dendritic cells and anti-Ig activated B cells as stimulators of the primary mixed leukocyte reaction. J. Exp. Med. 169:239-254.

40. Spittler, A., et al. 1995. IL-10 augments CD23 expression on U937 cells and down-regulates IL-4-driven CD23 expression on cultured human blood monocytes: effects of IL-10 and other cytokines on cell phenotype and phagocytosis. Immunology. 85:311-317.

41. Groux, H., Bigler, M., de Vries, J.E., and Roncarolo, M.G. 1998. Inhibitory and stimulatory effects of IL-10 on human $\mathrm{CD}^{+} \mathrm{T}$ cells. J. Immunol. 160:3188-3193

42. Kubin, M., Kamoun, M., and Trinchieri, G. 1994. Interleukin 12 syner- gizes with $\mathrm{B} 7 / \mathrm{CD} 28$ interaction in inducing efficient proliferation and cytokine production of human T cells. J. Exp. Med. 180:211-222.

43. Staunton, D.E., et al. 1989. A cell adhesion molecule, ICAM-1, is the major surface receptor for rhinoviruses. Cell. 56:849-853.

44. Greve, J.M., et al. 1989. The major human rhinovirus receptor is ICAM1. Cell. 56:839-847.

45. Gern, J.E., Vrtis, R., Kelly, E.A., Dick, E.C., and Busse, W.W. 1996. Rhinovirus produces nonspecific activation of lymphocytes through a monocyte-dependent mechanism. J. Immunol. 157:1605-1612.

46. Hofer, F., et al. 1994. Members of the low density lipoprotein receptor family mediate cell entry of a minor group common cold virus. Proc. Natl. Acad. Sci. USA. 91:1839-1842.

47. Durieu-Trautmann, O., Chaverot, N., Cazaubon, S., Strosberg, A.D., and Couraud, P.-O. 1994. Intercellular adhesion molecule 1 activation induces tyrosine phosphorylation of the cytoskeleton-associated protein cortactin in brain microvessel endothelial cells. J. Biol. Chem. 269:12536-12540.

48. Holland, J., and Owens, T. 1997. Signaling through intercellular adhesion molecule 1 (ICAM-1) in a B cell lymphoma line. The activation of lyn tyrosine kinase and the mitogen-activated protein kinase pathway. J. Biol. Chem. 272:9108-9112.

49. Duperray, A., et al. 1997. Molecular identification of novel fibrinogen binding site on the first domain of ICAM-1 regulation leukocyteendothelium bridging. J. Biol. Chem. 272:435-441.

50. Gardiner, E.E., and D'Souza, S.E. 1997. A mitogenic action for fibrinogen mediated through intercellular adhesion molecule-1. J. Biol. Chem. 272:15474-15480.

51. Casasnovas, J.M., and Springer, T.A. 1995. Kinetics and thermodynamics of virus binding to receptor. Studies with rhinovirus, intercellular adhesion molecule-1 (ICAM-1) and surface plasmon resonance. J. Biol. Chem. 270:13216-13224

52. Howard, M., and O'Garra, A. 1992. Biological properties of interleukin 10. Immunol. Today. 13:198-200

53. Moore, K.W., et al. 1990. Homology of cytokine synthesis inhibitory factor (IL-10) to the Epstein Barr virus gene BCRF1. Science. 248:1230-1234.

54. de Waal Malefyt, R., Abrams, J., Bennett, B., Figdor, C.G., and de Vries, J.E. 1991. Interleukin 10 (IL-10) inhibits cytokine synthesis by human monocytes: an autoregulatory role of IL-10 produced by monocytes. J. Exp. Med. 174:1209-1220.

55. Fiorentino, D.F., Zlotnik, A., Mosmann, T.R., Howard, M., and O'Garra, A. 1991. IL-10 inhibits cytokine production by activated macrophages. Immunol. 147:3815-3822

56. Kühn, R., Löhler, J., Rennick, D., Rajewski, K., and Müller, W. 1993. Interleukin-10 deficient mice develop chronic enterocolitis. Cell. 74:263-274.

57. Gerard, C., et al. 1993. Interleukin 10 reduces the release of tumor necrosis factor and prevents lethality in experimental endotoxemia. J. Exp. Med. 177:547-550 